\title{
Outcomes and prognostic contributors in patients with KRAS mutated non-small cell pulmonary adenocarcinomas: a single institution experience
}

\author{
Ethan A. Burns ${ }^{1}$, Joe E. Ensor ${ }^{1 \wedge}$, Jim Hsu $^{2}$, Jessica S. Thomas ${ }^{2}$, Randall J. Olsen ${ }^{2}$, Eric H. Bernicker ${ }^{1,3}$ \\ ${ }^{1}$ Houston Methodist Cancer Center, 6445 Main St. Outpatient Center, Houston, TX, USA; ${ }^{2}$ Department of Pathology and Genomic Medicine, \\ Houston Methodist hospital and Weill Cornell Medicine, Houston, TX, USA; ${ }^{3}$ Thoracic and Uveal Melanoma Oncology, Houston Methodist \\ Institute of Academic Medicine, Houston Methodist Cancer Center, 6445 Main St. Outpatient Center, Houston, TX, USA \\ Contributions: (I) Conception and design: EA Burns, JE Ensor, J Hsu, JS Thomas, RJ Olsen, EH Bernicker; (II) Administrative support: JS Thomas, \\ EH Bernicker; (III) Provision of study materials or patients: J Hsu, JS Thomas, RJ Olsen, EH Bernicker; (IV) Collection and assembly of data: EA \\ Burns, JE Ensor, EH Bernicker; (V) Data analysis and interpretation: EA Burns, JE Ensor, JS Thomas; (VI) Manuscript writing: All authors; (VII) \\ Final approval of manuscript: All authors.
}

Correspondence to: Eric H. Bernicker, MD. Houston Methodist Cancer Center, 6445 Main St. Outpatient Center, Floor 24. Houston, TX 77030, USA. Email: Bernicker@houstonmethodist.org.

Background: $K R A S$ is the most frequently encountered driver mutation in advanced non-small cell lung cancer (NSCLC). With targeted therapy for the most common KRAS mutation p.G12C on the horizon, the aim of this study is to retrospectively report outcomes in patients with KRAS mutated NSCLC.

Methods: This was a retrospective chart review of 7 hospitals in Texas with reflex biomarker testing in all lung adenocarcinomas. Patients were included if they had pathologically diagnosed adenocarcinoma of any stage originating in the lung with molecularly confirmed KRAS driver mutation of any genotypic subtype. Twelve-month survival was assessed and compared between KRAS p.G12C and all other detected KRAS mutations. Other outcomes including impact of age, sex, smoking status, and pack years smoked were assessed to determine if they had prognostic significance on mortality in KRAS mutated patients.

Results: There were 58 patients diagnosed with KRAS mutated NSCLC, $63.8 \%$ were at an advanced stage at diagnosis, $55.8 \%$ of patients were female, and $82.8 \%$ were white. The median age was 72 [52-88] years, and $93.1 \%$ were either current or prior smokers. KRAS p.G12C was the most common KRAS mutation (44.8\%). At diagnosis, patients with KRAS p.G12C had poorer performance statuses compared to other KRAS mutations. A total of $32(55.2 \%)$ patients died, 26 with advanced disease. In this study, current smoking status $(\mathrm{P}=0.1652)$, pack years smoked $(\mathrm{P}=0.6597)$, age $(\mathrm{P}=0.5092)$, sex $(\mathrm{P}=0.4309)$, and underlying KRAS codon mutation controlling for stage $(\mathrm{P}=0.2287)$ did not impact survival. However, KRAS p.G12C had a numerically lower 12 months overall survival (OS) compared to all other KRAS mutations in both early stage $(56.3 \%$ vs. $90.9 \%)$ and advanced stage $(25.0 \%$ vs. $47.6 \%)$ disease. Of note, $16(27.6 \%)$ patients had prior, concurrent, or second malignancies, but these did not significantly impact OS ( $\mathrm{P}=0.7696)$.

Conclusions: This study did not find a prognostic difference with sex, smoking history, age, or p.G12C mutation. The patients in this cohort with KRAS p.G12C had a numerically lower 12 -month overall survival in both early and advanced stage disease compared to other mutations, and over one-quarter had a notable history of previous and second primary malignancies.

Keywords: KRAS; non-small cell lung cancer (NSCLC); adenocarcinoma; smoking

^ ORCID: Ethan A. Burns, 0000-0002-8905-2039; Joe E. Ensor, 0000-0003-3533-4388. 
Submitted Mar 17, 2021. Accepted for publication Jun 10, 2021.

doi: $10.21037 /$ jtd-21-432

View this article at: https://dx.doi.org/10.21037/jtd-21-432

\section{Introduction}

Lung cancer is the leading cause of cancer diagnosis and cancer-related mortality world-wide. In the United States, an estimated 228,820 patients were diagnosed with lung cancer in the year 2020, and 135,720 individuals died (1). While current trends indicate long-term survival is improving in patients with local and regional disease, the majority of patients are diagnosed when their disease is widely metastatic, and outcomes for these patients remain poor with an estimated 5 -year overall survival (OS) of $2.9-10 \%(2-4)$. Despite these dismal statistics, survival over the past decade has improved. This likely reflects an evolving understanding of contributing genomic alterations and the emerging era of precision therapy targeting key driver mutations. Targeted therapy has dramatically transformed the therapeutic landscape and outcomes of non-small cell lung cancer (NSCLC), which comprises approximately $84 \%$ of lung cancer cases (1). Identifying NSCLC subtypes harboring driver mutations such as EGFR, RET, BRAF, ROS1, ALK, and NTRK have provided an avenue for targeted therapy and have proven useful for disease prognostication purposes.

Although KRAS is the most frequently encountered driver mutation in NSCLC, it has been considered an "undruggable" target, historically attributed to an absence of allosteric binding sites $(5,6)$. This carries important prognostic implications. Patients with KRAS mutations in comparison to patients with targetable driver mutations have a shorter median OS and disease-free survival (DFS) (7). Recently, a phase I study assessing the KRAS p.G12C inhibitory molecule Sotorasib (AMG 510) in patients with heavily pretreated NSCLC found an improved progression free survival (PFS) of 6.3 months, raising hope for a new line of therapy (8). With new treatment modalities on the horizon, identification and categorization of prognostic factors in the "real-world" clinical setting as well as the clinical trial population to allow for subgroup treatment comparisons is paramount in gaining a better understanding of patient outcomes. This study reports on outcomes and prognostic factors in patients with KRAS mutated adenocarcinoma in a standard of care clinical setting in Texas. The following article is reported in accordance with the STROBE reporting checklist (available at http://dx.doi. org/10.21037/jtd-21-432).

\section{Methods}

This study was conducted in accordance with the Declaration of Helsinki (as revised in 2013), was approved by the Houston Methodist institutional review board (IRB) at the study institution (IRB: PRO00017660:1), and individual consent for this analysis was waived.

This was a retrospective, observational study across 7 hospitals encompassing a single hospital network in Texas. Within this network, a reflex next-generation sequencing (NGS) panel has been implemented since 2017 on all newly diagnosed lung cancers, regardless of pathologic stage at time of diagnosis (9). A data review using the laboratory information system SoftPathDx (SCC) was used to query all cases of lung adenocarcinoma pathologically diagnosed between January 1, 2017 to January 1, 2019. Cases with the search terms "lung", "adenocarcinoma", and "MOLECULAR DIAGNOSTICS" in the interpretation or results free-text fields were included. Cases in which cytology did not mention "lung" were excluded. Each patient was then individually cross referenced in the electronic charting system EPIC to corroborate the clinicopathologic diagnosis and to obtain demographic and outcome characteristics. Patients that had a pathologic diagnosis within the hospital network but did not have a KRAS mutation, were diagnosed within the hospital system but treated elsewhere, or were treated within the hospital system but by a private provider that did not use the primary charting system EPIC were excluded from further analysis (Figure 1). All other patients with confirmed pulmonary adenocarcinoma with a KRAS mutation on tissue genomics were included. All patients that met criteria were retrospectively followed until death or last clinical visit up until 12/01/2020. At this institution, KRAS mutational testing was confirmed by one of two methodologies. The first modality was performed either as a single gene test using single base extension followed by mass spectrometry (OncoCarta panel and Sequenom MassARRAY instrument, Agena Biosciences). Alternatively, testing was performed as part of a solid organ tumor hotspot panel by next generation 


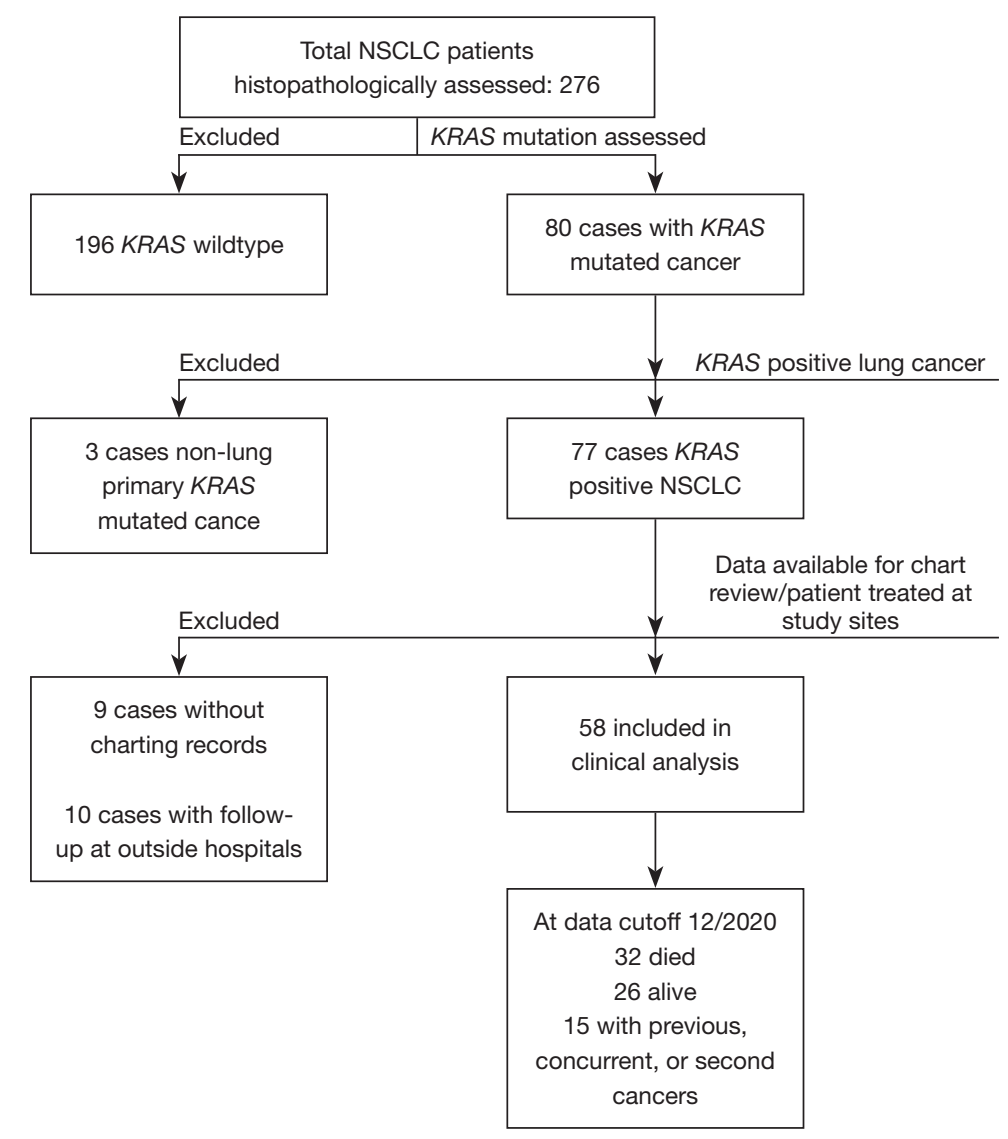

Figure 1 Consort Diagram with inclusion and exclusion parameters.

sequencing (Ion AmpliSeq Cancer Hotspot v2 and Ion Proton instrument, Life Technologies) on formalin fixed paraffin embedded (FFPE) tumor tissue by the performing Molecular Diagnostic Pathology Laboratory.

Patient information assessed included age at diagnosis with positive KRAS mutation, sex (male/female), ethnicity, smoking history (never smokers, previous smokers defined as total smoking cessation prior to the diagnosis of NSCLC, and current smokers defined as smoking $\geq 1$ tobacco containing product at the time of diagnosis), time from last cigarette use to diagnosis, pack years smoked, cancer stage (early, including stage I and II, and late, including III and IV) at time of KRAS diagnosis, performance status [measured by Eastern Cooperative Oncology Group (ECOG) from $0-5$, with 0 - being fully active and 5 being dead], specific $K R A S$ missense mutation site, presence of co-mutations (EGFR, ALK, STK11, TP53, etc.), PD-L1 activity, treatments used (including immunotherapy, chemotherapy, targeted therapy, radiation therapy, surgical lobectomy) and cycles of systemic therapy used. Previous, concurrent, or second cancers, median PFS and OS were also reported. Patients were followed from date of KRAS mutation confirmed to either date of death or last documented note in EPIC.

\section{Statistical analysis}

Descriptive statistics were reported as median with ranges for continuous variables and as frequencies and percentages for categorical variables. Patients were stratified as early stage (stage I and II) and advanced stage (stage III and IV), and KRAS driver mutation (p.G12C and all others). Mortality at 12 months was assessed and compared for prognostic risks including age, sex, type of KRAS mutation (KRAS p.G12C and all other KRAS mutations), pack years smoked, smoking status (current, former, or never smoker), time from last cigarette to diagnosis, and history of, or current diagnosis of another cancer. Comparisons of these prognostic factors were applied to 12 -month survival and were compared by using a Fisher's exact test (significance 
when the $\mathrm{P}$ value is $<0.05)$. A univariate logistic regression model was fitted to the 12-month survival data for age, pack years, and time elapsed since last smoked and odds ratios with $95 \%$ confidence interval (CI) were reported, and significance determined by a Wald Chi squared test (significance when the $\mathrm{P}$ value is $<0.05$ ). Total OS was further assessed for $K R A S$ mutations, smoking history, and sex by Kaplan-Meier methodology. If data was missing for subgroup analysis (i.e., total pack years) but pertinent patient information including KRAS mutation, date of diagnosis, and either date of last follow-up or date of death reported, were still included in the final analysis. If the patient was lost to follow-up, their last progress note was recorded as their last follow-up date.

\section{Results}

Between January 1, 2017 to January 1, 2019, a total of 276 cases of NSCLC were identified, of which there were $77(27.9 \%)$ cases of KRAS mutated NSCLC. Of these, $58(21.0 \%)$ patients with KRAS mutated NSCLC were available for clinical assessment and followed retrospectively until 12/1/2020 (Figure 1, Table 1). Figure 2 includes the proportion of $K R A S$ mutations for all pathologically confirmed cases (Figure $2 A$ ) and only patients included in the clinical analysis (Figure $2 B$ ). From the time of pathologically confirmed diagnosis, all patients in the clinical analysis were followed for a median time of 11.48 $(0.5-49.15)$ months. A summary of treatments is included in Table S1.

There were 32 (55.2\%) females and $26(44.8 \%)$ males, with a median age of 72 [52-88] years. The majority (48, $82.7 \%$ ) of patients were white. Patients had a median of 40 [0.2-120] pack years smoking history, and 32.5 [2-100] and $49.7(0.2-120)$ pack years with early and advanced stages, respectively. Of these, $79.3 \%$ of patients had $\geq 15$ pack year smoking history (Table 1). There were four $(6.7 \%)$ patients that had never smoked, two diagnosed at an early stage and two diagnosed at an advanced stage. A total of 13 (22.4\%) patients were smoking at the time of diagnosis, 10 of which had late-stage disease. Median time from last cigarette smoked to diagnosis was $22(0.5-42)$ and $10(0.25-50)$ years for early and advanced stage cancers, respectively. There were $5(8.6 \%)$ patients with missing smoking pack year data, and 4 (6.9\%) patients with missing data for time of last cigarette smoked. All other data was available for analysis. In terms of treatment, 21 (36.2\%) had surgery, 29 $(50.0 \%)$ had radiation, $30(51.7 \%)$ had chemotherapy, and
$22(37.9 \%)$ received immunotherapy. The most common chemotherapy regimen was carboplatin/pemetrexed, used with or without pembrolizumab in a total of $19(32.7 \%)$ patients. Of the 22 that received immunotherapy, 18 $(31.0 \%)$ were in combination with a systemic chemotherapy regimen. A total of $6(10.3 \%)$ patients received more than one line of systemic chemotherapy (Tables 1,2, Table S1).

\section{Patient outcomes}

At the time of data cutoff, 29 (50.0\%) patients had radiologically confirmed disease progression, (7 with early stage and 22 with late stage), 32 (55.2\%) patients died, and $16(27.6 \%)$ patients had either a previously diagnosed, concurrent, or second malignancy (Table S2, Figure S1). The median OS for those that died was $3.48(0.53-27.93)$ months. Disease progression was reported in 7 (33.3\%) patients with early-stage disease, with a median PFS of 9.1 (1.2-16.1) months, and 22 (59.4\%) patients with latestage disease, with a median PFS of 2.7 (0.6-5.6) months. Ten patients died prior to staging workup to determine radiographic evidence of progression. For the patients that died, $25(78.1 \%)$ deaths were attributed to disease disease progression and 7 (21.2\%) due to other causes including cardiac arrest [2], pneumonia [2], hemorrhagic pericardial effusion [1], respiratory failure [1] and unknown causes [1] (Table 1, Table S3).

All 10 patients with early stage $K R A S$ p.G12C mutated NSCLC received treatment ranging from chemotherapy/ immunotherapy, radiation, surgery, or a combination of the three. Similarly, all 11 patients with other KRAS mutations and early-stage disease received similar treatment modalities. The median ECOG performance status for earlystage disease was $1(0-1)$ for p.G12C patients and $0(0-1)$ for all other KRAS mutations. When assessing systemic therapy utilization for advanced disease, 8 (50.0\%) with $K R A S$ p.G12C and $4(19.0 \%)$ of patients with other KRAS mutations did not receive therapy. ECOG PS for advanced $K R A S$ p.G12C mutated disease and all other KRAS mutations was 2 [1-4] and 1 [1-3], respectively (Table 2, Table S1).

At 12 months, OS for KRAS p.G12C vs. all other KRAS mutations with early-stage disease was $56.3 \%$ versus $90.9 \%$, and for advanced stage disease was $25.0 \%$ versus $47.6 \%$, respectively. While the HR was 1.54 (95\% CI, 0.76, 3.1), this was not statistically significant $(\mathrm{P}=0.2287)$. Advanced stage compared to early stage was prognostically significant, with a hazard ratio (HR) of 3.228 [95\% CI, 1.32, 7.89, ( $\mathrm{P}=0.0102)]$. Assessment of prognostic factors including age, sex, pack 
Table 1 Baseline patient characteristics

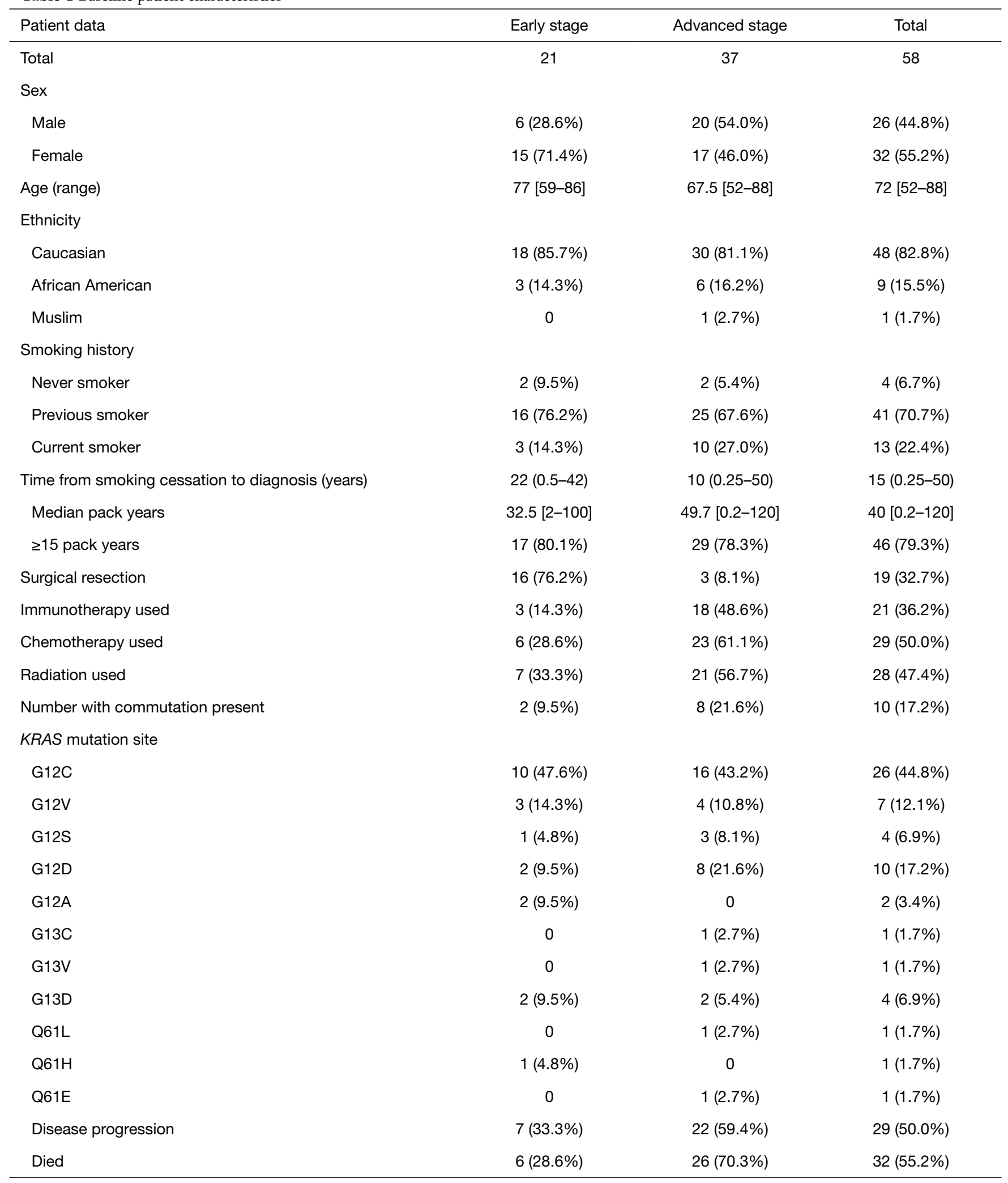



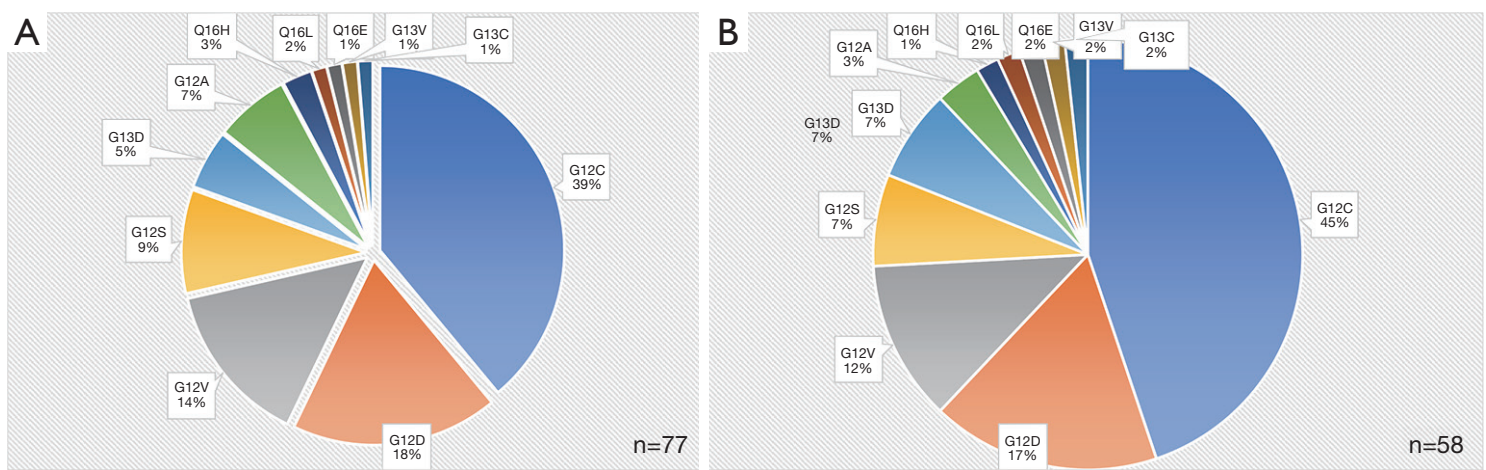

Figure 2 Proportion of specific KRAS mutations identified in all pathologic specimens and clinical cases assessed in this study. (A) Proportion based off the total number of pathologically confirmed cases. (B) Proportion based on clinical cases included in the final analysis.

Table 2 Characteristics of patients with KRAS p.G12C and all other KRAS mutations

\begin{tabular}{|c|c|c|}
\hline Characteristics & KRAS G12C & Other KRAS mutations \\
\hline Total & 26 & 32 \\
\hline Age & $70.5[55-86]$ & $73.5[54-88]$ \\
\hline \multicolumn{3}{|l|}{ Sex } \\
\hline Male & 7 & 19 \\
\hline Pack years smoked & $45.0(5-120)$ & $35(0.2-100)$ \\
\hline Current smoker & 8 & 7 \\
\hline Previous smoker & 17 & 23 \\
\hline Time from smoking cessation to diagnosis (years) & $10.0(0.25-40)$ & $15(0-50)$ \\
\hline Advance stage (III-IV) & 16 & 21 \\
\hline \multicolumn{3}{|l|}{ Performance status } \\
\hline Early stage & $1(0-1)$ & $0(0-1)$ \\
\hline Advanced stage & $2[1-4]$ & $1[1-3]$ \\
\hline CNS metastasis & 8 & 6 \\
\hline Other cancers & 10 & 6 \\
\hline Number with progression & 9 & 20 \\
\hline Deceased within 12 months & 16 & 12 \\
\hline
\end{tabular}

years smoked, current smoking status, KRAS mutation (p.G12C vs. all others), and other malignancies found no significant association (Table 3). The estimated probability of survival at 1 year for p.G12C was $38.1 \%$ (95\% CI, $20.0 \%$, $56.1 \%$ ) and $62.5 \%$ for all other mutations, but this was not significant $(\mathrm{P}=0.3183)$ (Figure $3 A)$. The estimated 12-month probability of survival for current smokers was $46.2 \%$ (95\% CI, $19.2 \%, 69.4 \%)$, for former smokers was $56.0 \%$ (95\% CI, $39.6 \%, 69.6 \%)$, and for the 4 never smokers was $25.0 \%$ (95\% CI, $0.9 \%, 66.5 \%$ ) (Figure 3B), but this was not significant 
Table 3 Prognostic variables for patients still alive by 12 months and dead within 12 months of follow-up

\begin{tabular}{|c|c|c|c|c|}
\hline Characteristics & Alive at 12 months & Died by 12 months & $\mathrm{OR} / \mathrm{HR}^{\star}(95 \% \mathrm{Cl})$ & $P$ value \\
\hline Sex & & & - & 0.4309 \\
\hline Male & 15 & 12 & & \\
\hline Female & 15 & 16 & & \\
\hline Smoking status & & & - & 0.1652 \\
\hline Current smoker & 6 & 6 & & \\
\hline Previous smoker & 23 & 19 & & \\
\hline Never smoker & 1 & 3 & & \\
\hline Advanced stage & 15 & 23 & & \\
\hline KRAS mutation & & & $1.53(0.76,3.08)$ & 0.2287 \\
\hline \multicolumn{5}{|l|}{ p.G12C } \\
\hline Early & 5 & 5 & & \\
\hline Advanced & 4 & 12 & & \\
\hline \multicolumn{5}{|l|}{ All others } \\
\hline Early & 10 & 2 & & \\
\hline Advanced & 11 & 12 & & \\
\hline
\end{tabular}

*, HR reported with stage of cancer and stage of cancer controlled for KRAS p.G12C versus all other mutations. HR, hazard ratio; OR, odds ratio.

$(\mathrm{P}=0.1652)$. The estimated 12 -month probability of survival based of patient sex was $46.2 \%$ (95\% CI, 19.2\%, 69.6\%) for females and $25.0 \%$ (95\% CI, $8.95 \%, 66.5 \%)$ for males, which was not statistically significant $(\mathrm{P}=0.5487)$.

A total of $16(27.6 \%)$ had either a previous malignancy/ benign tumor or concurrently treated malignancy (Table 2). Three patients had more than two additional malignancies, one patient with breast cancer and meningioma (p.G12C), and one patient with prostate cancer and urothelial bladder cancer (p.G12C), and one patient with cervical and salivary gland tumor (p.G12D) (Table S2). History of malignancy did not significantly impact survival at 12 months $(\mathrm{P}=0.7696)$ (Table 3).

\section{Mutation analysis}

$K R A S$ p.G12C was the most frequently diagnosed driver mutation of the KRAS subtypes, occurring in 26 (44.8\%) patients in the clinical analysis and in $30(39.0 \%)$ of all pathologically diagnosed KRAS mutated NSCLC (Table 1, Figures 1-4). It occurred in 10 (17.2\%) patients with earlystage cancers and $16(27.6 \%)$ patients with advanced stage cancers. A total of $16(61.5 \%)$ patients with KRAS p.G12C mutated NSCLC died at the time of data cutoff, $5(31.2 \%)$ of which had early-stage disease. The median OS for patients with KRAS p.G12C and advanced disease $(\mathrm{n}=16)$ was $6.0(0.93-32.5)$ months, and for early-stage disease $(n=10)$ was 14.9 months $(0.67-49.2)$ months. The distribution of other mutations is summarized in Figure 1.

Ten patients $(17.2 \%)$ had at least one commutation reported (Table 1). These included a second KRAS mutation (p.V14) in one patient with a pre-existing p.G12D mutated NSCLC, Met Exon 14 (with p.G12V) (n=1), ARD1A (n=1), STK11 $(\mathrm{n}=1), \operatorname{TP} 53(\mathrm{n}=3), \operatorname{SMO}(\mathrm{n}=1)$, and GNA11 $(\mathrm{n}=1)$, BRAF G469A (n=1), ASXL1 (n=1). Of these commutations, 

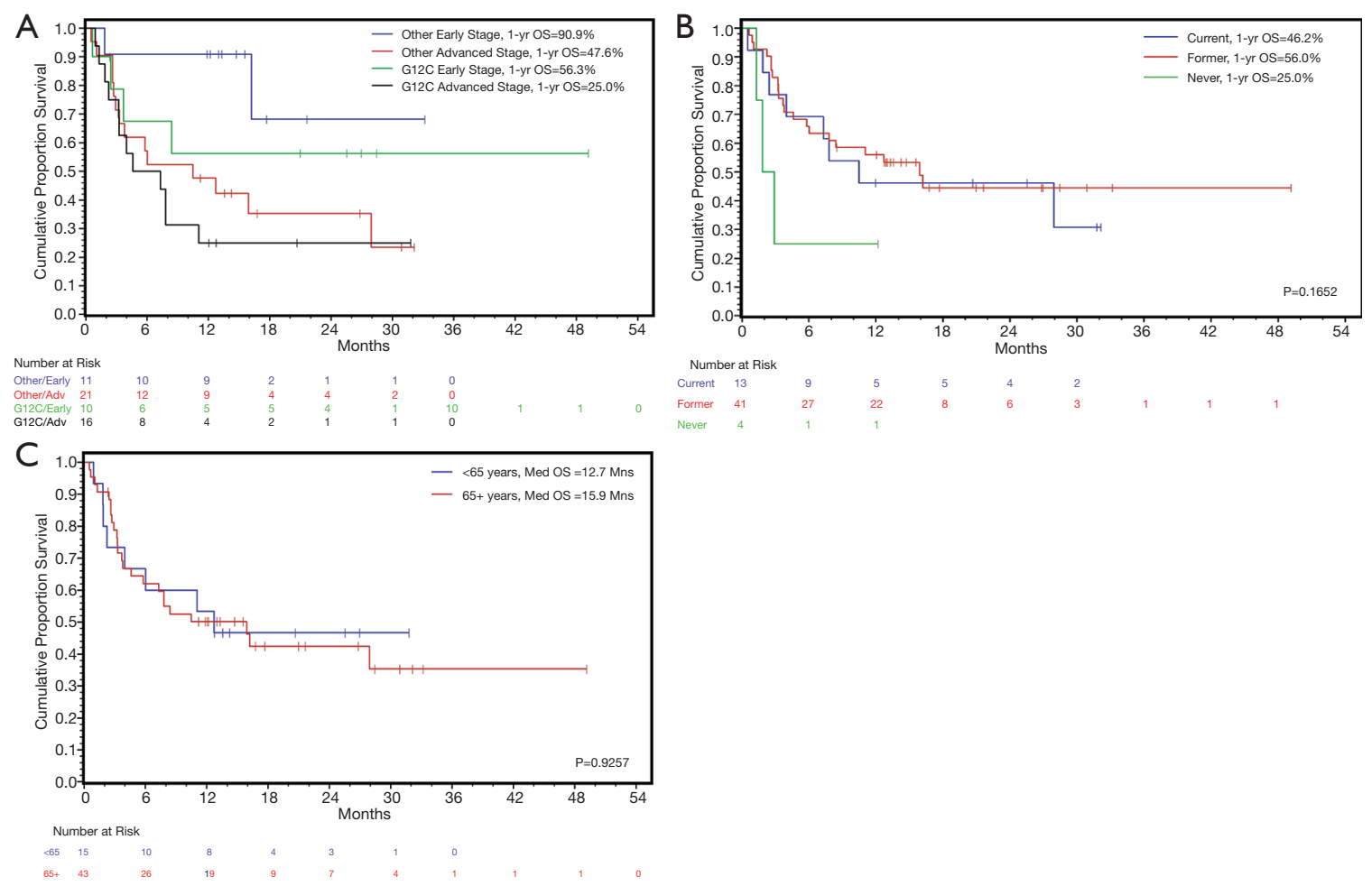

Figure 3 Kaplan-Meier estimates of patient overall survival. (A) Patient survival comparison between KRAS p.G12C, and all other mutations, controlling for early and advanced stage. (B) Patient survival based on smoking status. (C) Patient survival based on age.

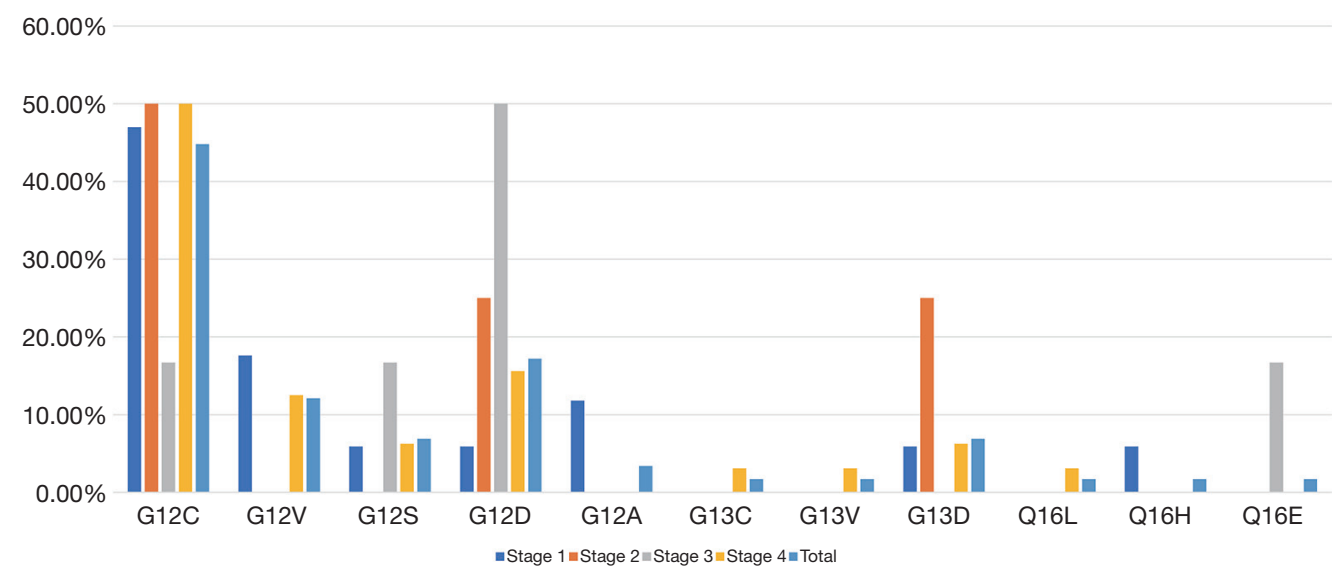

Figure 4 Percentage of clinically assessed patients with NSCLC harboring various KRAS mutations by tumor stage at diagnosis. NSCLC, non-small cell lung cancer.

3 patients (STK11, ARD1A, Met Exon 14), all with advanced disease, died in the 12 months following diagnosis.

\section{Discussion}

Over 3 decades have passed since the discovery of the KRAS oncogenic driver mutation in human lung cancers. Since then, knowledge on genotypic variations, role in disease and treatment prognostication, and patient outcomes continues to evolve (10). With the possibility of targeted therapy for the most common point mutation p.G12C entering the phase 1b/II CodeBreak 101 clinical trial (8, NCT04185883), this 
retrospective study sought to assess patient characteristics in a standard of care clinical setting involving 1 academic and 6 community hospital settings across Texas. In this patient cohort, KRAS driver mutations in non-small cell pulmonary adenocarcinomas occurred in $27.9 \%$ of reported lung cancer cases, with the most frequently detected mutation being $K R A S$ p.G12C. These mutations were mutually exclusive with other driver mutations, like other reports $(11,12)$. Most of the patient population had a smoking history, were older (median age of 72 years), white, with a slightly higher proportion of females $(55.2 \%)$, findings also consistent with other studies (13-15). This patient population predominately had advanced stage (III and IV) NSCLC at diagnosis, and $48.3 \%$ of the patient population died within 12 months following the diagnosis of KRAS mutated NSCLC. In general, patients with the KRAS p.G12C mutation had a poorer baseline ECOG performance status at diagnosis. While the survival differences were not statistically different, patients with early and advanced stage KRAS p.G12C had numerically lower survival at 12-month, which may reflect the poorer performance status at diagnosis impacting the ability of some of these patients to receive systemic therapy. It is also worth noting that the period covered in this study include broad use of immunotherapy, and these poor survival numbers underscore the unmet needs of improving therapies for $K R A S$ mutated lung cancers.

$K R A S$ mutations have demonstrated a poorer OS compared to KRAS wild type NSCLC. Studies have demonstrated that patients with KRAS mutated NSCLC have a shorter median OS regardless of the presence of commutations, shorter 2-year survival, and inferior outcomes when receiving chemotherapy in the metastatic setting $(14,16,17)$. When comparing specific codon mutations and specific genotypic point mutations, results on the impact on survival and PFS are variable. In a study with 677 patients with KRAS mutated advance stage NSCLC, patients with mutations in KRAS codon $13(\mathrm{n}=53)$ had inferior survival compared to codon $12(\mathrm{n}=624)$, with a 2 -month survival difference $(\mathrm{P}=0.008)$, when controlling for age, sex, and smoking status. Another analysis of 450 patients with KRAS mutated, metastatic pulmonary adenocarcinomas found no difference in OS between codon 12 and 13 mutations, although there was a numerically lower 2-year survival with codon 13 (14). While neither of these studies found a point mutation specific impact on survival (12), other studies have found genotypic differences in survival, with point mutations in p.G12C and p.G12V reported to have poorer survival compared to other $K R A S$-mutant subtypes (18). In a study of 75 Asian patients with advanced NSCLC, KRAS p.G12C portended a better PFS, and even more so when these patients were treated with pemetrexed based chemotherapy as a first line agent (19). In the present study, there was no difference in OS between KRAS p.G12C and all other $K R A S$ mutations. Due to the small sample size of this study, dichotomous comparison between codon 12 and codon 13 was not possible. Furthermore, this study primarily had white patients. Whether survival is different in other ethnic groups is uncertain and should be assessed in additional studies. While there was a numerically lower 12-month survival in $K R A S$ p.G12C mutations with advanced disease compared to all other mutations, these patients (both early and advanced stage) had a poorer performance status at diagnosis.

In this study, nearly $50 \%$ of patients died in the 12 months following diagnosis. Many of these patients had advanced disease, with only 5 deaths reported in early-stage disease. There is conflicting data regarding the prognostic implication of KRAS mutations in earlier stage lung cancers. Keohavong reported a nonsignificant, yet notable poorer survival in patients with $K R A S$ mutated stage 1 NSCLC (20). In addition, Nadal et al. found a poorer DFS survival in patients with $K R A S$ mutated lung cancers, with a notable decrease in DFS and OS in the patients with a KRAS p.G12C mutation (21). While Izar et al. also found that patients with KRAS mutated lung cancers had poor DFS and OS, results noted a superior DFS with mutations in codon 12 (22). Comparatively, Yu et al. and Shepherd et al. failed to find a difference in prognostic implications for early-stage disease $(12,23)$. In the present study, 21 patients had early-stage disease at diagnosis. Results did not suggest prognostic impacts on KRAS mutation and stage on survival or progression, but the small sample size precludes a larger analysis.

Determining whether all patients with early stage should be tested for $K R A S$ driver mutations is uncertain. While KRAS mutation testing as a stand-alone assay is not routinely recommended, it is considered reasonable to include as a component in larger assays (24). However, data shows that at an international level, physician ordered molecular testing is still lacking. Despite guidelines recommending routine $E G F R$ mutation testing at diagnosis of advance NSCLC, a 2019 study found that only $80 \%$ of oncologists ordered appropriate testing, and of these, $18 \%$ of clinicians did not wait for results prior to starting systemic therapy in part due to long turn-around time $(24,25)$. To circumnavigate these delays, the institution involved in the present study has standardized biomarker testing parameters and reflex ordered molecular biomarker 
testing for lung cancer patients, which includes analysis and reporting of EGFR, KRAS, BRAF, and ERBB2 gene mutations; MET exon 14 skipping; ALK, RET, and ROS1, NTRK1 and NTRK3 gene rearrangements; MET gene amplification; and PD-L1 expression by immunohistochemistry. Implementing this reflex ordered molecular testing strategy in patients with newly diagnosed lung adenocarcinomas has significantly improved turn-around times and allowed for a more uniform and consistent analysis of NSCLC for targetable mutations (9), which may be beneficial in future analyses of the impact of KRAS mutations in early stage, resectable NSCLC. Furthermore, the recent approval of the EGFR inhibitor (T790M, L858R, and exon 19 deletion) osimertinib as an adjuvant treatment for early stage resected adenocarcinoma following results from the phase III ADAURA trial (26) may bring broader next generation sequencing into earlier stages of disease.

Results from this study did not find a significant survival difference between current/former/never smokers harboring the KRAS oncogenic driver mutation. While studies have reported superior OS for never-smokers compared to current or former smokers $(27,28)$, this difference may be due to a lack of homogenous oncogenic mutation subgroups, with KRAS more frequent in smokers, and EGFR and $A L K$ mutations more common in never smokers $(12,28)$. However, when controlling for the specific oncogene, there has been no difference in survival between current/former/never smoking status $(12,28)$. Results in this patient population appear to support this data as well. In addition, the small proportion of never smokers (6.8\%), current/former smokers (93.2\%), and median pack years smoked is like other reported studies in patients with advanced KRAS mutated NSCLC $(12,29)$. In this study, $93.2 \%$ of patients were current/former smokers, and $79.3 \%$ of patients that had ever smoked had greater than 15 pack years. Another study in patients with advanced NSCLC had significantly poorer OS when they had smoked greater than 15 pack years compared to less than 15 pack years $(0.30,95 \% \mathrm{CI}, 1.12-1.51, \mathrm{P}<0.001)$ or were never smokers (OR 1.57, 95\% CI, 1.36-1.80, $\mathrm{P}<0.001$ ). This study only included advanced stage NSCLC, and had a large proportion of never smokers, which also included patients who smoked $<100$ cigarettes (27). The KRAS study population in the present study was heterogenous, including patients of all stages of cancer, and only 4 patients were never smokers. Furthermore, patients that had ever smoked $<100$ lifetime cigarettes were considered smokers rather than never smokers, and so this combined with the relatively small sample size make comparison of smoking status on survival challenging, and may have contributed to lack of survival difference at 12 months with respect to pack years and smoking status.

There is limited knowledge regarding second malignancies in KRAS mutated NSCLC. In this study, $27.6 \%$ of patients had an additional primary cancer, and $50 \%$ of these were seen in early-stage NSCLC. Most of these cancers occurred prior to the diagnosis of NSCLC. Studies have found that patients with KRAS mutated early lung cancers have a higher risk of developing second malignancies, ranging from $1.5-15 \%$, and most commonly second primary lung cancers, particularly in patients that smoke $(23,30)$. Shepherd et al. found that patients with KRAS mutated NSCLC had a nearly 3 -fold increase in the risk of developing second primary malignancies, whereas patients that received adjuvant chemotherapy had a lower risk of a second primary malignancy (23). Patients in the present study had a range of prior malignancies, including cancers of the genitourinary tract, colorectal cancer, leukemia, lymphoma, and breast cancer, as well as benign tumors that impact patient quality of life (Table S2). While this small patient cohort precludes an in-depth analysis, further observational studies of second KRAS mutated lung cancer in patients with known prior cancers and smoking history are needed. Furthermore, given the potential risk for second primaries in KRAS mutated NSCLC, especially in early-stage patients, it is possible that screening for second primary tumors in this patient population is warranted.

This was a retrospective analysis and as a result has inherent limitations. As a chart review, evaluations are limited to what is provider reported and as such is not always inclusive of variables such as current performance status, smoking history, or date of death. In several cases, the patients were known to have entered hospice care, but a date of death cannot be determined. In addition, 19 patients did not receive treatment at the study institution after primary diagnosis and were not included for additional treatment analysis and outcomes. This, combined with the low baseline sample size impacted the analysis on survival, prognostic variables impacting mortality, and differences in variables when controlling for age and KRAS mutation. As a result, while there was a large numerical difference in mortality for patients with KRAS p.G12C mutations compared to other KRAS mutations in this cohort, no statistical difference in this subgroup or other subgroups was found. Furthermore, the small sample size limited the ability to perform a multivariate analysis controlling for 
$K R A S$ mutations on all outcomes.

\section{Conclusions}

With the evolving era of precision medicine now finding targeted therapy for KRAS p.G12C mutated lung cancers, further studies to determine outcomes in this patient population are needed. This analysis found that patients with $K R A S$ p.G12C mutations had a non-significant poorer OS at 12 months, and that KRAS mutated patients in this cohort had a high proportion of second malignancies. Additional studies to further assess the risk and implication of additional malignancies, as well as prognostic risk factors in standard of care setting are needed.

\section{Acknowledgments}

Funding: None.

\section{Footnote}

Reporting Checklist: The authors have completed the STROBE reporting checklist. Available at http://dx.doi. org/10.21037/jtd-21-432

Data Sharing Statement: Available at http://dx.doi. org/10.21037/jtd-21-432

Peer Review File: Available at http://dx.doi.org/10.21037/jtd$21-432$

Conflicts of Interest: All authors have completed the ICMJE uniform disclosure form (available at http://dx.doi. org/10.21037/jtd-21-432). Dr. EHB serves as an unpaid editorial board member of Fournal of Thoracic Disease from Feb 2019 to Jan 2023. Dr. EHB reports receiving personal fees from Novartis, Pfizer, AstraZeneca, Blueprint medicine and Guardant Health, outside of the submitted work. The other authors have no conflicts of interest to declare.

Ethical Statement: The authors are accountable for all aspects of the work in ensuring that questions related to the accuracy or integrity of any part of the work are appropriately investigated and resolved. This study was conducted in accordance with the Declaration of Helsinki (as revised in 2013), was approved by the Houston Methodist institutional review board (IRB) at the study institution (IRB: PRO00017660:1), and individual consent for this retrospective analysis was waived.

Open Access Statement: This is an Open Access article distributed in accordance with the Creative Commons Attribution-NonCommercial-NoDerivs 4.0 International License (CC BY-NC-ND 4.0), which permits the noncommercial replication and distribution of the article with the strict proviso that no changes or edits are made and the original work is properly cited (including links to both the formal publication through the relevant DOI and the license). See: https://creativecommons.org/licenses/by-nc-nd/4.0/.

\section{References}

1. Lung Cancer-Non-Small Cell: Statistics. Available online: https://www.cancer.net/cancer-types/lungcancer-non-small-cell/statistics. Date Last Accessed: 1 December, 2020.

2. Lu T, Yang X, Huang Y, et al. Trends in the incidence, treatment, and survival of patients with lung cancer in the last four decades. Cancer Manag Res 2019;11:943-53.

3. Wang T, Nelson RA, Bogardus A, et al. Five-year lung cancer survival: which advanced stage nonsmall cell lung cancer patients attain long-term survival? Cancer 2010;116:1518-25.

4. Román M, Baraibar I, López I, et al. KRAS oncogene in non-small cell lung cancer: clinical perspectives on the treatment of an old target. Mol Cancer 2018;17:33.

5. Cox AD, Der CJ. Ras history: The saga continues. Small GTPases 2010;1:2-27.

6. Cox AD, Fesik SW, Kimmelman AC, et al. Drugging the undruggable RAS: Mission possible? Nat Rev Drug Discov 2014;13:828-51.

7. Kris MG, Johnson BE, Berry LD, et al. Using multiplexed assays of oncogenic drivers in lung cancers to select targeted drugs. JAMA 2014;311:1998-2006.

8. Hong DS, Fakih MG, Strickler JH, et al. KRASG12C Inhibition with Sotorasib in Advanced Solid Tumors. N Engl J Med 2020;383:1207-17.

9. Anand K, Phung TL, Bernicker EH, et al. Clinical Utility of Reflex Ordered Testing for Molecular Biomarkers in Lung Adenocarcinoma. Clin Lung Cancer 2020;21:437-42.

10. Rodenhuis S, van de Wetering ML, Mooi WJ, et al. Mutational activation of the K-ras oncogene. A possible pathogenetic factor in adenocarcinoma of the lung. $\mathrm{N}$ Engl J Med 1987;317:929-35.

11. Biernacka A, Tsongalis PD, Peterson JD, et al. The potential utility of re-mining results of somatic mutation 
testing: KRAS status in lung adenocarcinoma. Cancer Genet 2016;209:195-8.

12. Yu HA, Sima CS, Shen R, et al. Prognostic impact of KRAS mutation subtypes in 677 patients with metastatic lung adenocarcinomas. J Thorac Oncol 2015;10:431-7.

13. Mao C, Qiu LX, Liao RY, et al. KRAS mutations and resistance to EGFR-TKIs treatment in patients with nonsmall cell lung cancer: a meta-analysis of 22 studies. Lung Cancer 2010;69:272-8.

14. El Osta B, Behera M, Kim S, et al. Characteristics and Outcomes of Patients With Metastatic KRAS-Mutant Lung Adenocarcinomas: The Lung Cancer Mutation Consortium Experience. J Thorac Oncol 2019;14:876-89.

15. Riely GJ, Kris MG, Rosenbaum D, et al. Frequency and distinctive spectrum of KRAS mutations in never smokers with lung adenocarcinoma. Clin Cancer Res 2008;14:5731-4.

16. Mascaux C, Iannino N, Martin B, et al. The role of RAS oncogene in survival of patients with lung cancer: a systematic review of the literature with meta-analysis. Br J Cancer 2005;92:131-9.

17. Rodenhuis S, Slebos RJ. The ras oncogenes in human lung cancer. Am Rev Respir Dis 1990;142:S27-30.

18. Ihle NT, Byers LA, Kim ES, et al. Effect of KRAS oncogene substitutions on protein behavior: implications for signaling and clinical outcome. J Natl Cancer Inst 2012;104:228-39.

19. Lei L, Wang WX, Yu ZY, et al. A Real-World Study in Advanced Non-Small Cell Lung Cancer with KRAS Mutations. Transl Oncol 2020;13:329-35.

20. Keohavong P, DeMichele MA, Melacrinos AC, et al. Detection of K-ras mutations in lung carcinomas: relationship to prognosis. Clin Cancer Res 1996;2:411-8.

21. Nadal E, Chen G, Prensner JR, et al. KRAS-G12C mutation is associated with poor outcome in surgically resected lung adenocarcinoma. J Thorac Oncol 2014;9:1513-22.

22. Izar B, Zhou H, Heist RS, et al. The prognostic impact

Cite this article as: Burns EA, Ensor JE, Hsu J, Thomas JS, Olsen RJ, Bernicker EH. Outcomes and prognostic contributors in patients with KRAS mutated non-small cell pulmonary adenocarcinomas: a single institution experience. $\mathrm{J}$ Thorac Dis 2021;13(8):4785-4796. doi: 10.21037/jtd-21-432 of KRAS, its codon and amino acid specific mutations, on survival in resected stage I lung adenocarcinoma. J Thorac Oncol 2014;9:1363-9.

23. Shepherd FA, Domerg C, Hainaut P, et al. Pooled analysis of the prognostic and predictive effects of KRAS mutation status and KRAS mutation subtype in early-stage resected non-small-cell lung cancer in four trials of adjuvant chemotherapy. J Clin Oncol 2013;31:2173-81.

24. Kalemkerian GP, Narula N, Kennedy EB, et al. Molecular Testing Guideline for the Selection of Patients With Lung Cancer for Treatment With Targeted Tyrosine Kinase Inhibitors: American Society of Clinical Oncology Endorsement of the College of American Pathologists/ International Association for the Study of Lung Cancer/ Association for Molecular Pathology Clinical Practice Guideline Update. J Clin Oncol 2018;36:911-9.

25. Peters M, Kim ES, Hirsch V. Clinical Use of Epidermal Growth Factor Receptor Testing in Patients With Advanced Lung Cancer by Physicians: Survey of US and International Patterns. J Glob Oncol 2019;5:1-7.

26. Wu YL, Tsuboi M, He J, et al. Osimertinib in Resected EGFR-Mutated Non-Small-Cell Lung Cancer. N Engl J Med 2020;383:1711-23.

27. Janjigian YY, McDonnell K, Kris MG, et al. Pack-years of cigarette smoking as a prognostic factor in patients with stage IIIB/IV nonsmall cell lung cancer. Cancer 2010;116:670-5.

28. Paik PK, Johnson ML, D'Angelo SP, et al. Driver mutations determine survival in smokers and neversmokers with stage IIIB/IV lung adenocarcinomas. Cancer 2012;118:5840-7.

29. Sousa CC, Pinto J, Martins N, et al. Characterization of Patients with KRAS-Mutated Advanced Non-Small Cell Lung Cancer. Eur Respir J 2019;54:PA4684.

30. Rice D, Kim HW, Sabichi A, et al. The risk of second primary tumors after resection of stage I nonsmall cell lung cancer. Ann Thorac Surg 2003;76:1001-7; discussion 1007-8. 


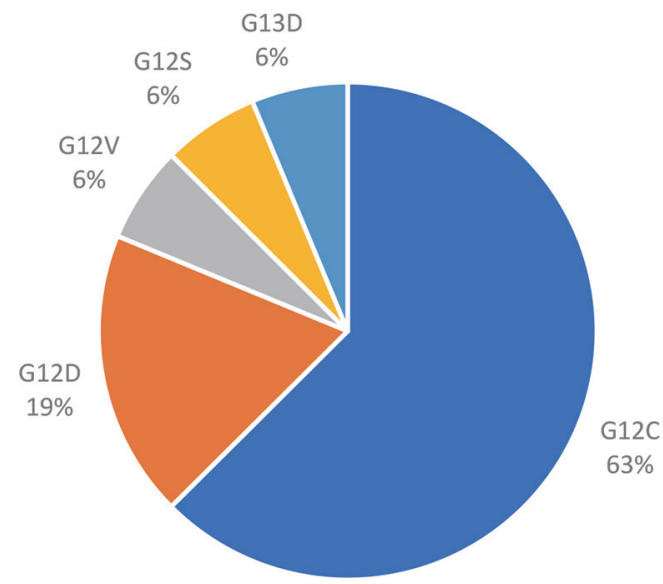

Figure S1 Percent of patients with history of additional malignancies based on KRAS mutation profile. Patients with previous or concurrent malignancies were seen in KRAS G12C, G12D, G12V, G12S, G13D driver mutations.

Table S1 Summary of treatments for each KRAS driver mutation

\begin{tabular}{|c|c|c|c|c|c|}
\hline $\begin{array}{l}\text { KRAS Mutation } \\
\text { (Stage at Diagnosis) }\end{array}$ & $\begin{array}{l}\text { Chemotherapy Regimens } \\
\text { +/-combined Immunotherapy } \\
\text { (cycles) }\end{array}$ & $\begin{array}{l}\text { Immunotherapy Regimens } \\
\text { (monotherapy) (cycles) }\end{array}$ & $\begin{array}{l}\text { Mean cycle of } \\
\text { systemic therapy }\end{array}$ & $\begin{array}{l}\text { Radiation } \\
\text { Therapy }\end{array}$ & $\begin{array}{l}\text { Surgical } \\
\text { Resection }\end{array}$ \\
\hline G12C (I) & - & - & & Yes & Yes \\
\hline G12C (I) & - & - & & Yes & - \\
\hline G12C (I) & $\begin{array}{l}\text { Carboplatin/ Pemetrexed/ } \\
\text { Pembrolizumab }\end{array}$ & - & 1 & - & Yes \\
\hline G12C (I) & $\begin{array}{l}\text { Carboplatin/ Pemetrexed/ } \\
\text { Pembrolizumab }\end{array}$ & - & & - & Yes \\
\hline G12C (I) & - & - & & - & Yes \\
\hline G12C (I) & - & - & & - & Yes \\
\hline G12C (I) & - & - & & - & Yes \\
\hline G12C (I) & - & - & & - & - \\
\hline G12C (II) & Carboplatin/ Paclitaxel & - & 6 & Yes & - \\
\hline G12C (II) & Carboplatin/ Pemetrexed ${ }^{a}$ & - & 4 & - & Yes \\
\hline G12C (IV) ${ }^{\alpha}$ & $\begin{array}{l}\text { Bevacizumab/ Carboplatin/ } \\
\text { Paclitaxel; Navelbine/ } \\
\text { Gemcitabine }\end{array}$ & - & $6 / 4$ & - & Yes \\
\hline G12C (IV) & Avastin & - & Unknown & - & Yes \\
\hline G12C (IV) & $\begin{array}{l}\text { Carboplatin/ Pemetrexed/ } \\
\text { Pembrolizumab }\end{array}$ & - & 1 & - & Yes \\
\hline G12C (IV) & $\begin{array}{l}\text { Carboplatin/ Pemetrexed/ } \\
\text { Pembrolizumab }\end{array}$ & - & 1 & - & Yes \\
\hline G12C (IV) & $\begin{array}{l}\text { Carboplatin/ Pemetrexed/ } \\
\text { Pembrolizumab; Pemetrexed/ } \\
\text { Carboplatin }\end{array}$ & - & $3 / 3$ & - & Yes \\
\hline
\end{tabular}

Table S1 (continued) 
Table S1 (continued)

\begin{tabular}{|c|c|c|c|c|c|}
\hline $\begin{array}{l}\text { KRAS Mutation } \\
\text { (Stage at Diagnosis) }\end{array}$ & $\begin{array}{l}\text { Chemotherapy Regimens } \\
+ \text { /-combined Immunotherapy } \\
\text { (cycles) }\end{array}$ & $\begin{array}{l}\text { Immunotherapy Regimens } \\
\text { (monotherapy) (cycles) }\end{array}$ & $\begin{array}{c}\text { Mean cycle of } \\
\text { systemic therapy }\end{array}$ & $\begin{array}{l}\text { Radiation } \\
\text { Therapy }\end{array}$ & $\begin{array}{l}\text { Surgical } \\
\text { Resection }\end{array}$ \\
\hline G12C (IV) & - & Pembrolizumab & 2 & - & Yes \\
\hline G12C (IV) & - & - & - & - & Yes \\
\hline G12C (IV) & - & - & - & - & - \\
\hline G12C (IV) & $\begin{array}{l}\text { Carboplatin/ Pemetrexed/ } \\
\text { Pembrolizumab; Pemetrexed/ } \\
\text { Pembrolizumab }\end{array}$ & - & $4 / 5$ & - & - \\
\hline G12C (IV) & - & - & - & - & - \\
\hline G12C (IV) & - & - & - & - & - \\
\hline G12C (IV) & - & - & - & - & - \\
\hline G12V (I) & - & - & - & - & Yes \\
\hline G12V (I) & $\begin{array}{l}\text { Carboplatin/ Pemetrexed/ } \\
\text { Pembrolizumab }\end{array}$ & - & Unknown & - & Yes \\
\hline G12V (I) & - & - & - & - & Yes \\
\hline G12V (IV) ${ }^{\beta}$ & Carboplatin/ Paclitaxel & - & 5 & Yes & - \\
\hline G12S (IV) & $\begin{array}{l}\text { Carboplatin/ Pemetrexed/ } \\
\text { Pembrolizumab }\end{array}$ & Pembrolizumab (13) & 4 & Yes & - \\
\hline G12S (IV) & - & - & - & Yes & - \\
\hline G12D (I) & - & - & - & Yes & Yes \\
\hline G12D (II) $)^{*}$ & - & $\begin{array}{l}\text { Pembrolizumab; Nivolumab } \\
\qquad(15 ; 3)\end{array}$ & - & Yes & Yes \\
\hline G12D (III) & $\begin{array}{l}\text { Carboplatin/ Paclitaxel/ } \\
\text { Pembrolizumab }\end{array}$ & - & & Yes & - \\
\hline G12D (III) ${ }^{\mathrm{a}}$ & Cisplatin/ Venorelbine & - & 4 & Yes & Yes \\
\hline G12D (III) & Carboplatin/ Pemetrexed & - & 4 & Yes & Yes \\
\hline G12D (IV) ${ }^{a}$ & Cisplatin/ Gemcitabine & Pembrolizumab (4) & 3 & - & Yes \\
\hline
\end{tabular}

Table S1 (continued) 
Table S1 (continued)

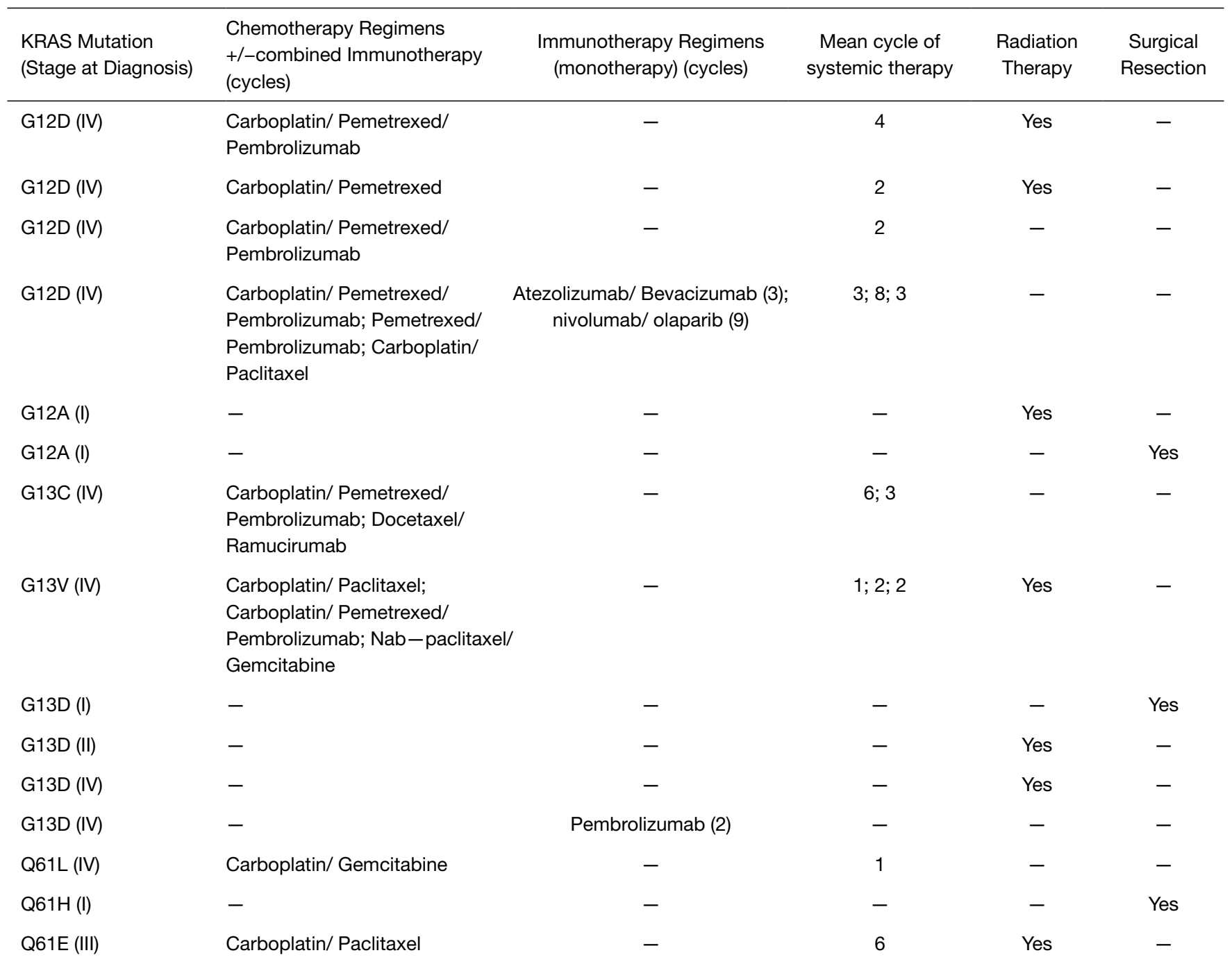

${ }^{a}$ : Adjuvant chemotherapy+/-immunotherapy; ${ }^{b}$ : could not tolerate Durvalumab after 2 cycles, switched to Pembrolizumab; ${ }^{\alpha}$ : Previous Lobectomy and adjuvant chemotherapy in 2004 with cisplatin/etoposide, and carboplatin/pemetrexed in 2014 for recurrence. Recurrence

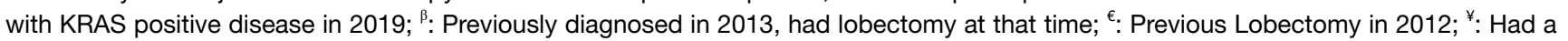
second KRAS V141 mutation with metastatic recurrence. Also received oncolytic viral injection as part of an ongoing clinical trial. 
Table S2 Specific second malignancies

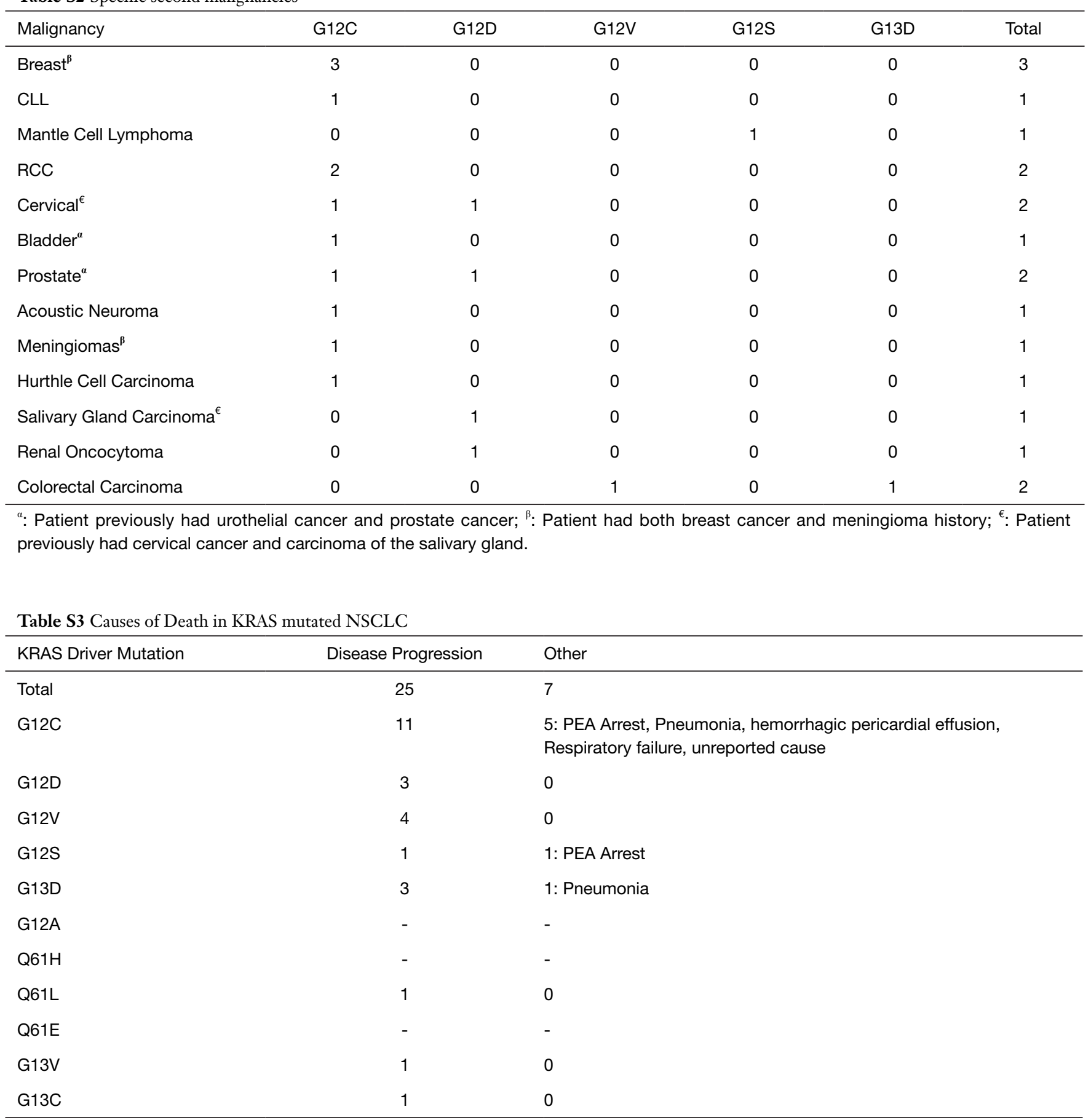

PEA: Pulseless Electrical Activity. 